\title{
Anteroposterior and mediolateral postural adaptations during single and duals tasks in healthy young adults
}

\author{
Martín G. Rosario a, ${ }^{*}\left(\mathbb{D}\right.$, Aleena Jose a ${ }^{(0)}$ \\ a Physical Therapy Program, Texas Woman's University, Dallas Campus; Texas \\ *Corresponding author Email: mrosario1@twu.edu ; Telephone: 241-689-6500 \\ DOI: https://doi.org/10.34256/ijpefs2139
}

Received: 28-08-2021, Revised: 14-09-2021; Accepted: 18-09-2021; Published: 24-09-2021

\begin{abstract}
Dual tasks are fundamental and standard for daily walking and balance movements. However, further research is required to determine the comprehensive postural profile during challenging dual cognitive tasks. To distinguish the influence of dual cognitive tasks on anterior-posterior (AP) and mediolateral (ML) jerk (direction of sway), velocity, and distance in young adults with normal balance systems. Nineteen subjects took part in this inquiry ( 2 males and 17 females), with a mean age of $23.9+\backslash-2.3$ years. The participants were instrumented using a lumbar accelerometer and a dynamometer designed to capture sway. All subjects completed eight balance tests comprising four single and four dual-cognitive tasks involving counting backward by three, starting at the number 100 (dual-task). Postural modifications were prominent in the AP direction, with a faster jerk, velocity, and considerable distance than in the ML direction. The introduction to challenging balance situations, including dual tasks, provoke AP direction adaptations to preserve balance through variations in AP parameters, indicating the engagement of the sensory reweighting system.
\end{abstract}

Keywords: Anteroposterior, Movements, Postural adaptations

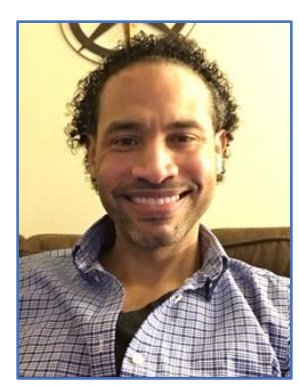

Martin G. Rosario PT, Ph.D., CSFI, ATRIC. Obtained his Ph.D. in Neuroanatomy from the University of Puerto Rico Medical Sciences Campus. Master in Physical Therapy from the University of Puerto Rico Medical Sciences Campus School of Physical Therapy. Bachelor in Biology with a Major in Human Focus from University of Puerto Rico Bayamon Campus. Currently serves as an Assistant Professor at Texas Woman's University (TWU) School of Physical Therapy Dallas, Clinical Researcher at an HIV community clinic La Perla de Gran Precio in Puerto Rico, and Director/Coordinator of Motor Control Research Laboratory at TWU Dalla.

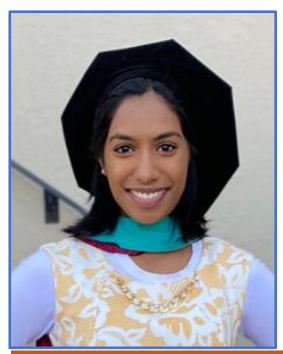

Aleena Jose, DPT, PT. Has a DPT from Texas Woman's University (TWU). Bachelor of Science in kinesiology from TWU.Currently involved in six published journal articles focusing on temporo-spatial gait adaptations in Latino-Hispanic adults with controlled type II diabetes, promoting health awareness in the general public, and neuromuscular timing activity of ankle musculature in young adults during altered sensory systems.

\section{Introduction}

Balance entails combining different systems to maintain postural control, such as visual, vestibular, and proprioceptive systems. All these networks communicate with one another to adapt to different environments and challenges. Balance is the body's ability to sustain its center of mass over the base of support [1]. After injuries or normal aging processes, the balance systems decline, and the accuracy to maintain equilibrium is reduced; therefore, instability or falls are the following events [1, 2]. Furthermore, deterioration in cognitive function is related to a gradual reduction of the gray matter located in the frontal and temporal lobes, starting at the age of 20 [3]. Among the factors related to cognitive function and interplay, worth contemplating is the association with postural control. Typically, when attention is 
shifted towards the cognitive area, less awareness is paid to the balance network, and as a result, sway increases $[4,5]$. Unfortunately, with the aging process, the effectiveness of postural control diminishes with dual tasking, often resulting in instability and falls [3].

In a review by $\mathrm{Li}$ et al., various challenging scenarios combined with dual tasks could provoke an increase in postural sway, such as unstable surfaces and decreased sensory input (darkroom or eyes closed) [6]. Furthermore, the authors illustrated how complex cognitive tasks play a more significant role and provoke considerable modifications in dynamic situations compared to single tasks [6]. Contrarily, other studies have discovered conflicting evidence, with equivocal or improved balance performance in dual tasks compared to single tasks [7-10]. Regardless, various studies have determined that multi-balance activities with cognitive components are beneficial for improving balance during dual cognitive tasks $[8,11]$.

Limited studies have analyzed how other balance parameters, such as sway in the anteriorposterior $\mathrm{AP}$ and mediolateral (ML) directions, are affected by dual cognitive tasks. Understanding jerk (direction of sway), velocity (speed of sway), and distance of sway in the AP and ML directions across various balance conditions could provide clinicians with a quantitative measure of postural control. We consider this previous remark to be crucial because otherwise would be undetectable while performing balance outcome measures in healthy young adults. Previous studies have identified increased sway and neuromuscular activation in the AP direction compared to the $\mathrm{ML}$ direction during strenuous balance tasks in young, healthy subjects $[12,13]$. In contrast, $M L$ instability has been noted in populations with musculoskeletal impairments, such as low back pain and muscle weakness. Similarly, ML instability is linked to impairments related to neuromuscular origins, such as stroke, and even age-related changes in balance control [14 - 16]. Although investigators have accepted that healthy individuals with intact balance mechanisms maintain a quiet stance in the AP direction, investigators have accepted that healthy individuals with intact balance mechanisms maintain a quiet stance. We believe that research on the extent to which there is AP instability during complex tasks or increased dual-task costs in this population is still limited.

Considering the aforementioned, we aim to comprehend the impact of introducing dual cognitive tasks following single tasks, in contrast to $\mathrm{AP}$ and $\mathrm{ML}$ jerk (direction of sway), velocity, and distance in young adults with healthy balance systems.

\section{Methods}

The Texas Women's University IRB approved this study (protocol \#20092). We recruited participants via word-of-mouth from research assistants at the Texas Woman's University Health Science Center in Dallas. First, a research associate explained each partaker's role in the investigation and obtained written informed consent. Subsequently, subjective screening was performed to acquire the participants' demographic information, such as weight, height, and age.

This study sought young, healthy adults aged range-18-45 years. The exclusion criteria were established to avert any confounding factors that could alter posture and, therefore, data collection. The exclusion criteria were (1) intake of drugs that cause drowsiness 24 hours before participation, such as some allergy meds, any surgeries or injuries at the back or lower extremity in the past six months, and (3) demonstrating significant stability problems or inability to sustain balance for 30 seconds during the Romberg test.

\subsection{Instruments and balance protocol}

Balance was assessed using the MobilityLab (accelerometer and gyroscope) protocols. A MobilityLab sensor was placed on each participant at the level of the third lumbar vertebra. Participants were first instructed to maintain a static bipedal stance on a firm surface (practice tasks) while concomitantly focusing their gaze on an orange square attached to the wall 10-feet away. Next, eight balance tests were performed on a foam pad (2.4. height, 15.5 in. long, 12.5 in. wide) while repeating the previous static stance and focusing on the orange square. The eight balance tests consisted of four single and four dualcognitive tasks requiring counting backward by three, starting at number 100 (dual-task). Each balance task was intended to challenge the sensory input related to postural balance. For instance, when eyes are closed on a foam task, the visual input is canceled, and proprioceptive feedback is altered. The addition of head movements further challenges stability by altering vestibular input.

The four single task conditions on foam were: 1 ) eyes open (EO), 2) eyes closed (EC), 3) eyes open with the head moving up and down following a 
metronome set to $60 \mathrm{bpm}(\mathrm{EOH})$, and 4) eyes closed with the head moving in the vertical direction following a cadence of $60 \mathrm{bpm}(\mathrm{ECH})$.

The four cognitive dual-task tests on foam were as follows: 1) eyes open counting backward $(E O C), 2)$ eyes closed counting backward (ECC), 3) eyes open counting backward while moving the head up and down at a cadence of $60 \mathrm{bpm}(\mathrm{EOCH})$; and 4) eyes closed counting backward while moving the head up and down at a cadence of $60 \mathrm{bpm}(\mathrm{ECCH})$.

Each test was recorded for $15 \mathrm{~s}$, and the variables of interest included jerk (direction of sway) in the mediolateral (ML) and anterior-posterior (AP) directions, as well as the velocity and distance in the $\mathrm{AP}$ and $\mathrm{ML}$ directions.

\subsection{Data Analysis}

The data collected were inputted into the SPSS Data Analysis 25 system, and analysis of variance was performed. The variables of interest in this inquiry were sway, jerk, and velocity during each condition. In addition, differences were examined within each variable comparing $\mathrm{AP}$ and $\mathrm{ML}$ during the different protocols, such as deviations in sway across single-task conditions, and similar comparisons were performed for cognitive tasks. This inquiry considers a P-value of 0.05 , or less significant.

\section{Results}

Table 1 shows the participants' demographic characteristics.

Table 1 Demographic Information

\begin{tabular}{|l|l|}
\hline Characteristics & Study Participants $n=19$ \\
\hline Gender & Male $=2 ;$ Female $=17$ \\
\hline Age & $23.9 \pm 2.3$ years \\
\hline Height (inches) & M=65.4 \pm 3.3 \\
\hline Weight (pounds) & $139.9 \pm 22.4$ \\
\hline
\end{tabular}

A total of 19 subjects participated in this study ( 2 males and 17 females), with a mean age of $23.9+\backslash$ 2.3 years. All 19 participants performed a single-task balance assessment. Later in the study, the same group of subjects completed the cognitive tasks. All participants were considered healthy with no underlying diseases and had stable vital signs, including blood pressure, heart rate, and pulse oximetry, indicating good cardiovascular health.

Table 2 shows comparisons of the AP jerk and $M L$ jerk during the single tasks. A significant difference in $\mathrm{AP}(0.05 \pm-0.03)$ and $\mathrm{ML}$ jerk $(0.10+/-0.005)$ during ECH was noted $(p=0.001)$. The AP and ML jerk for EOHUDCOG $(0.14+/-0.14$ and $0.02+/-0.02$ respectively) and ECHUDCOG $(0.12+/-0.13$ and $0.04+/-0.04$ respectively) tasks also yield significant variance with a $p$-value of 0.01 . Figure 1 illustrates the AP jerk and ML jerk during single and dual tasks, with distinctly quicker postural adaptations in the ML direction compared to AP.

Table 3 compares the AP and ML velocities during single and dual tasks. Significant variance in AP $(0.17+/-0.14)$ and $\mathrm{ML}(0.05+/-0.03)$ velocity was identified during the EOH task $(p=0.001)$. Moreover, the AP velocity during ECCOG, EOHUDCOG, and ECHUDCOG were remarkably higher than the ML velocity $(p=0.01)$. The corresponding figure 2 shows how the AP velocity was faster than the ML velocity, although quick postural adaptations were evident in both directions across both single and dual tasks.

Table 4 shows the AP and ML distances during single and dual tasks. AP distance was notably higher than $\mathrm{ML}$ distance during various tasks, with a $\mathrm{p}$-value of 0.01 for ECH, ECCOG, and ECHUDCOG and a pvalue of 0.001 for $E O H$ and ECHUDCOG. Figure 3 displays increased directional distance as the complexity of the task increases, although it is more evident in the AP direction.

\section{Discussion}

The purpose of this investigation was to determine the impact of dual cognitive tasks on balance by comparing jerk, velocity, and distance in the AP and ML directions in young adults with healthy sensory systems. Our results are deficient when categorizing which balance task had the most significant variance in AP and ML parameters because fluctuations in jerk, velocity, and distance were inconsistent across the tasks. However, even after participants adapted to the challenges of the single tasks, considerable differences in AP and $\mathrm{ML}$ parameters were observed during $\mathrm{ECH}$ and cognitive tasks such as EOHCOG and ECHCOG. Postural adaptations were apparent in both the $\mathrm{AP}$ and $\mathrm{ML}$ directions, but were more altered in the AP direction, with a quick jerk, faster velocity, and greater distance than the ML direction. 
Table 2 ANOVA comparing AP Jerk and ML Jerk variables during single and dual tasks on a foam surface. A p-value $\leq$ of 0.01 is considered to be significant.

\begin{tabular}{|l|l|l|l|}
\hline $\begin{array}{c}\text { Single Task } \\
\text { N= 19 }\end{array}$ & \multicolumn{1}{|c|}{ AP JERK Means and SD } & \multicolumn{1}{|c|}{ ML JERK Means and SD } & P-Value \\
\hline EO: & $0.05+/-0.05$ & $0.09+/-0.22$ & 0.37 \\
\hline EC: & $0.07+/-0.14$ & $0.06+/-0.12$ & 0.77 \\
\hline EOH & $0.05+/-0.03$ & $0.10+/-0.005$ & 0.001 \\
\hline ECH & $0.12+/-0.14$ & $0.03+/-0.04$ & 0.05 \\
\hline EOCOG & $0.07+/-0.07$ & $0.03+/-0.04$ & 0.05 \\
\hline ECCOG & $0.04+/-0.03$ & $0.03+/-0.03$ & 0.30 \\
\hline EOHUDCOG & $0.14+/-0.14$ & $0.02+/-0.02$ & 0.01 \\
\hline ECHUDCOG & $0.12+/-0.13$ & $0.04+/-0.04$ & 0.01 \\
\hline $\begin{array}{l}\text { EO: Eyes Open, EC: Eyes Closed, EOH: eyes open with vertical head movement, ECH: eyes closed with } \\
\text { vertical head movement, AP:anterior-posterior, ML: mediolateral, S.D.=Standard deviation }\end{array}$ \\
\hline
\end{tabular}

Table 3 ANOVA comparing AP VEL and ML VEL variables during single and dual tasks on a foam surface. A p-value $\leq$ of 0.01 is considered to be significant.

\begin{tabular}{|l|l|l|l|}
\hline $\begin{array}{c}\text { Single Task } \\
\mathbf{N = 1 9}\end{array}$ & AP VEL Means and SD & ML VEL Means and SD & P-Value \\
\hline EO: & $0.15+/-0.11$ & $0.07+/-0.04$ & 0.05 \\
\hline EC: & $0.16+/-0.14$ & $0.09+/-0.08$ & 0.05 \\
\hline EOH & $0.17+/-0.14$ & $0.05+/-0.03$ & 0.001 \\
\hline ECH & $0.21+/-0.20$ & $0.06+/-0.03$ & 0.05 \\
\hline EOCOG & $0.17+/-0.14$ & $0.07+/-0.04$ & 0.05 \\
\hline ECCOG & $0.17+/-0.09$ & $0.06+/-0.05$ & 0.01 \\
\hline EOHUDCOG & $0.19+/-0.17$ & $0.07+/-0.03$ & 0.01 \\
\hline ECHUDCOG & $0.16+/-0.10$ & $0.08+/-0.03$ & 0.01 \\
\hline $\begin{array}{l}\text { EO: Eyes Open, EC: Eyes Closed, EOH: eyes open with vertical head movement, } \\
\text { ECH: eyes closed with vertical head movement, AP:anterior-posterior, ML: } \\
\text { mediolateral, S.D. }=\text { Standard deviation }\end{array}$ \\
\hline
\end{tabular}


Table 4 ANOVA comparing AP DIST and ML DIST variables during single and dual tasks on a foam surface. A p-value $\leq$ of 0.01 is considered to be significant.

\begin{tabular}{|l|l|l|l|}
\hline \multicolumn{1}{|c|}{$\begin{array}{c}\text { Single Task } \\
\text { N= 19 }\end{array}$} & \multicolumn{1}{|c|}{ AP DIST Means and SD } & \multicolumn{1}{|c|}{ ML DIST Means and SD } & \multicolumn{1}{c|}{ P-Value } \\
\hline EO: & $0.07+/-0.04$ & $0.06+/-0.03$ & 0.20 \\
\hline EC: & $0.10+/-0.05$ & $0.06+/-0.03$ & 0.05 \\
\hline EOH & $0.12+/-0.04$ & $0.04+/-0.01$ & 0.001 \\
\hline ECH & $0.15+/-0.06$ & $0.07+/-0.03$ & 0.01 \\
\hline EOCOG & $0.10+/-0.06$ & $0.06+/-0.02$ & 0.05 \\
\hline ECCOG & $0.11+/-0.04$ & $0.06+/-0.02$ & 0.01 \\
\hline EOHUDCOG & $0.16+/-0.07$ & $0.06+/-0.01$ & 0.01 \\
\hline ECHUDCOG & $0.15+/-0.6$ & $0.07+/-0.1$ & 0.001 \\
\hline $\begin{array}{l}\text { EO: Eyes Open, EC: Eyes Closed, EOH: eyes open with vertical head movement, ECH: eyes closed with } \\
\text { vertical head movement, AP:anterior-posterior, ML: mediolateral, S.D.=Standard deviation }\end{array}$ \\
\hline
\end{tabular}
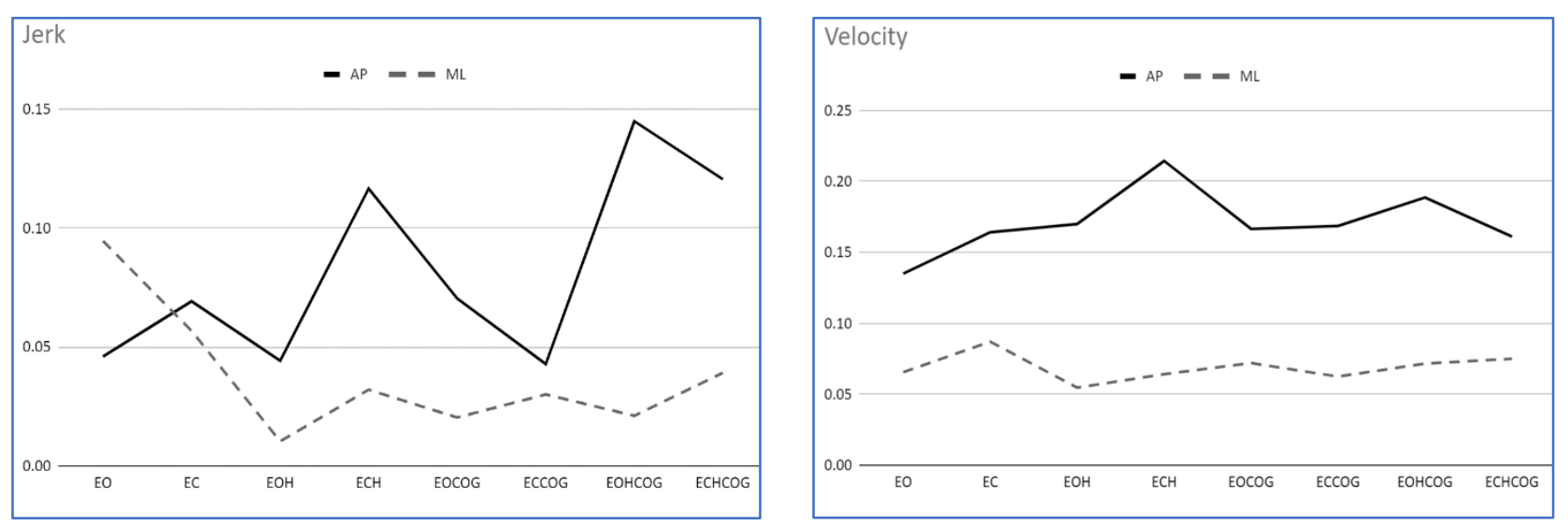

Figure 1 and Figure 2 shows the comparison of AP Jerk and ML Jerk variables and AP and ML velocities variables during single and dual tasks

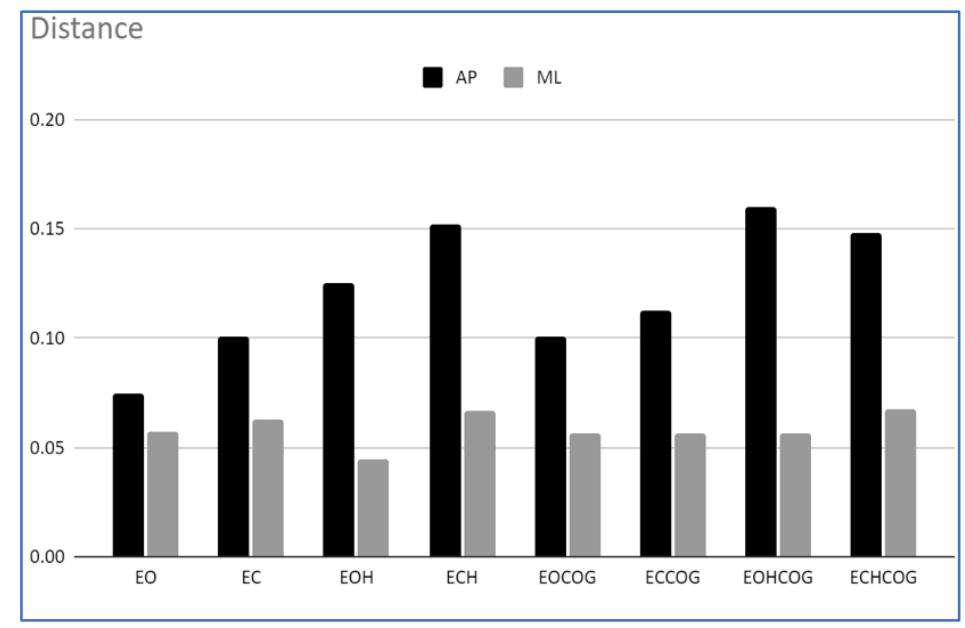

Figure 3 Comparison of AP and ML distance variables during single and dual tasks. 
Although none of the participants adapted to the stepping strategy, indicating effective balance networks, fluctuations in AP sway across tasks can be attributed to the participant's acquaintance performing the single tasks before the dual cognitive task and difficulty with sensory reweighting during challenging situations. Most of the variations occurring in the AP direction also indicate that AP is the primary route for balance stabilization during quiet stance.

Our first findings demonstrate more sway in the AP direction than in the ML direction, particularly with tasks that challenge the sensory systems simultaneously and individually. The increase in AP sway during the ECH task can be attributed to the novelty of the task and visual dependence for postural control. Additionally, the summation of head movements and cognitive tasks further elicits postural instability, resulting in higher AP sway during the EOHCOG task. These findings substantiate the previous literature that increased AP sway during balance tasks, altering multiple sensory systems [12]. Interestingly, Vincent and Vuillerme revealed decreased AP displacement and no considerable deviations in ML displacement in young adults during dual cognitive tasks involving subtractions due to increased stiffness in the ankle musculature for postural control [17]. An increase in ML sway during complex balance tasks is often associated with low back pain, hip dysfunction, weak hip muscles, stroke, Parkinson's disease, and elderly populations $[12,16,18]$. ML instability was undetectable in our participants, considering that they were relatively young, healthy, and had no history of the aforementioned health conditions. However, incremental fluctuations in AP sway across challenging balance conditions indicate recruitment of the ankle strategy, inability to stiffen the ankle musculature, and increased dual-task interference. Figure 1 also reflects the ANOVA results for AP and ML jerk, with quicker adjustments in $\mathrm{ML}$ jerk compared to AP during conditions such as ECH and EOHCOG, further proving AP instability during complex tasks in our participants.

The second primary outcome of this study illustrates that, similar to AP jerk characteristics, AP velocity was also considerably higher than $\mathrm{ML}$ velocity across dual tasks (ECCOG, EOHUDCOG, ECHUDCOG). The EOH task also exhibited markedly higher AP velocity, which is plausible because of the unfamiliarity of balance stabilization with head movements. However, this deviation in AP velocity subsided during the dual tasks, as illustrated in Figure 2, signifying that the participants became accustomed to the balance condition. During the single tasks, AP velocity during
ECCOG, EOHUDCOG, and ECHUDCOG were not higher than $\mathrm{EOH}$ because participants already had experience performing the balance conditions. Thus, our results align with previous findings, with increased AP sway velocity during dual tasks, although a unipedal stance was performed in this study [19]. Nonetheless, AP velocity was lower in ECHUDCOG than in the more demanding EOHUDCOG task, which could also be due to acclimation. Therefore, future studies should consider performing conditions in a random order to avoid the carryover of balance mechanisms established during single tasks.

The third main finding of this research shows that in contrast to variances in jerk and velocity across tasks in the AP and ML directions, differences in AP and $\mathrm{ML}$ distance were apparent in more conditions, such as EOH, ECH, ECCOG, EOHUDCOG, and ECHUDCOG tasks. Similar to Figure 2, these differences in distance did not progressively increase as the complexity of the task increased (see Figure 3). While ML distance was relatively invariant across tasks, AP distance increased, for the most part, to maintain static balance during challenging conditions, especially during the EOHCOG task. Likewise, a study comparing COP in soccer athletes and non-athletes derived more significant displacement in the center of pressure and AP velocity in the non-athletic group, with increased $M L$ instability during stringent balance conditions [20]. However, we were unaware of whether our participants were athletic.

Furthermore, our experiments share some similarities with the query by Lanzarin et al., where increased body oscillations during cognitive tasks were noted [4]. Conversely, other investigations identified decreased postural sway during dual cognitive activities compared to single tasks in healthy young adults [10, 21]. Thus, based on the initial results, alterations in AP parameters during different balance conditions suggest difficulty in allocating attention to dual tasks and diminished anticipatory postural responses.

To understand why the AP direction is usually the way to adapt to balance conditions, balance mechanisms need to be deciphered. The ankle strategy is an automatic postural response initiated to maintain balance, where a small external perturbation displaces the center of mass (COM). The hip strategy was initiated when COM displacements were more prominent and faster. Multiple inquiries support that displacements in the AP direction are regulated by ankle strategy and displacements in the ML by hip strategy $[1,22]$. We suspect that our balance

Int. J. Phys. Educ. Fit. Sports, 10(3) (2021), 84-91 | 89 
conditions were not rigorous enough to elicit a hip strategy. Thus, our subjects primarily used the ankle strategy for balance equilibrium, which concurs with previous findings of low to medium balance difficulty tasks compensated with ankle strategy [23]. AP instability in our participants indicated cognitive cost in young, healthy adults with healthy balance systems. These findings support the establishment of postural stability exercises in young, healthy adults to refine balance mechanisms - skills that can persist into older adulthood, potentially decreasing fall risk. Older

The limitations of the current study include the inability to standardize the step width and ankle position for various tasks. For example, those with postural instability in the frontal plane compensate by increasing step width [18]. Additionally, gender differences were unaccounted; previous queries have recognized female participants to have lower balance stability due to higher COM, COP, and sway, requiring higher neuromuscular efforts to stabilize posture [12]. Thus, subsequent inquiries should account for ankle positions and gender differences when analyzing balance factors.

\section{Conclusion}

The purpose of this study was to understand the impact of dual cognitive tasks on standing balance, such as total sway, sway direction (anterior-posterior and medio-lateral), and velocity in young adults with healthy sensory systems. This study establishes a baseline balance profile that depicts similarities in postural control during single and dual cognitive tasks among multiple scenarios in young adults. Overall, adaptations to challenging balance conditions were primarily employed in the AP direction to maintain balance, although fluctuations in AP parameters indicate difficulty with sensory reweighting. We imply that introducing balance training in young, healthy adults could enhance balance mechanisms that can be carried over into adulthood. Further studies are needed to identify whether adding cognitive tasks while challenging the sensory system during dynamic tasks, such as gait, considerably alters balance responses. Additionally, we encourage researchers to further assess neuromuscular adaptations during dual cognitive balance and postural changes during dualmotor tasks.

\section{References}

[1] A. Shumway-Cook \& M.H. Woollacott. Motor control theory and practical applications,
Lippincott Williams \& Wilkins. Edition, 2nd edition, (2001) 176-177.

[2] G. Yogev-Seligmann, J.M. Hausdorff, \& N. Giladi, The role of executive function and attention in gait, Movement disorders : official journal of the Movement Disorder Society, 23(3) (2008) 329472. [DOI] [PubMed]

[3] N. Raz, K.M. Rodrigue, \& E.M. Haacke, Brain aging and its modifiers: insights from in vivo neuromorphometry and susceptibility weighted imaging, Annals of the New York Academy of Sciences, 1097 (2007) 84-93. [DOI] [PubMed]

[4] M. Lanzarin, P. Parizzoto, T. Libardoni, L. Sinhorim, G.M. Tavares, G.M. Santos, The influence of dual-tasking on postural control in young adults, Fisioterapia e Pesquisa, 22(1) (2015) 61-68. [DOI]

[5] N.B. Alexander, \& J.M. Hausdorff, Guest editorial: linking thinking, walking, and falling. The journals of gerontology, Series A, Biological sciences and medical sciences, 63(12) (2008) 1325-1328. [DOI] [PubMed]

[6] K. Li, L. Bherer, A. Mirelman, I. Maidan, \& J.M. Hausdorff, Cognitive Involvement in Balance, Gait and Dual-Tasking in Aging: A Focused Review From a Neuroscience of Aging Perspective, Frontiers in Neurology, 9 (2018) 913. [DOI] [PubMed]

[7] R. Kiss, D. Brueckner, \& T. Muehlbauer, Effects of Single Compared to Dual Task Practice on Learning a Dynamic Balance Task in Young Adults, Frontiers in Psychology, 9 (2018) 311. [DOI] [PubMed]

[8] T.A. Worden \& L.A. Vallis, Concurrent performance of a cognitive and dynamic obstacle avoidance task: influence of dual-task training, Journal of Motor Behavior, 46(5) (2014) 357368. [DOI] [PubMed]

[9] L.M. Ross, J. Register-Mihalik, J.P. Mihalik, K. Mcculloch, W.E. Prentice, E.W. Shields, K.M. Guskiewicz, Effects of single-task versus a dualtask paradigm on cognition and balance in healthy subjects, Journal of Sport Rehabilitation, 20(3) (2011) 296-310. [DOI] [PubMed]

[10] K.Z.H. Li, E. Roudaia, M. Lussier, L. Bherer, A. Leroux, P. A. McKinley, Benefits of cognitive dual-task training on balance performance in healthy older adults, The Journals of Gerontology: Series A, 65A(12) (2010) 13441352. [DOI] [PubMed]

[11] J.W. Błaszczyk, M. Beck, \& D. Sadowska, Assessment of postural stability in young healthy 
subjects based on directional features of posturographic data: vision and gender effects, Acta neurobiologiae experimentalis, 74(4) (2014) 433-442. [PubMed]

[12] F.A. Barbieri, T. Penedo, L. Simieli, R.A. Barbieri, A.M. Zagatto, J.H. van Diëen, M. Pijnappels, S.T. Rodrigues, \& P.F. Polastri, Effects of Ankle Muscle Fatigue and Visual Behavior on Postural Sway in Young Adults, Frontiers in Physiology, 10 (2019) 643. [DOI] [PubMed]

[13] M. Bergamin, S. Gobbo, T. Zanotto, J. C. Sieverdes, C.L. Alberton, M. Zaccaria, \& A. Ermolao, Influence of age on postural sway during different dual-task conditions, Frontiers in Aging Neuroscience, 6 (2014) 271. [DOI] [PubMed]

[14] W. Yodchaisarn, R. Puntumetakul, A. Emasithi, R. Boucaut, \& U. Chatchawan, Altered postural sway during quiet standing in women with clinical lumbar instability, Journal of Physical Therapy Science, 30(8) (2018) 1099-1102. [DOI] [PubMed]

[15] E. Yu, M. Abe, K. Masani, N. Kawashima, F. Eto, N. Haga, \& K. Nakazawa, Evaluation of postural control in quiet standing using center of mass acceleration: comparison among the young, the elderly, and people with stroke, Archives of Physical Medicine and Rehabilitation, 89(6) (2008) 1133-1139. [DOI]

[16] N. Vuillerme, \& H. Vincent, How performing a mental arithmetic task modify the regulation of centre of foot pressure displacements during bipedal quiet standing, Experimental Brain Research, 169(1) (2006) 130-134. [DOI]

[17] R. Miyachi, T. Abiko, H. Nakano, K. Shiraiwa, A. Goda, J. Miyazaki, S. Murata, Relationship between frontal-plane gait parameters and balance tests in older adults, Archives of Physical Health and Sports Medicine 4(1) (2021) 01-06.

[18] E.J. Bisson, D. McEwen, Y. Lajoie, M. Bilodeau. Effects of ankle and hip muscle fatigue on postural sway and attentional demands during unipedal stance, Gait Posture, 33(1) (2011) 837. [DOI] [PubMed]

[19] L.A. Thompson, M. Badache, S. Cale, L. Behera, \& N. Zhang, Balance Performance as Observed by Center-of-Pressure Parameter Characteristics in Male Soccer Athletes and Non-Athletes, Sports (Basel, Switzerland), 5(4) (2017) 86. [DOI] [PubMed]
[20] MC. Hunter \& M.A. Hoffman, Postural control: visual and cognitive manipulations, Gait Posture, 13(1) (2001) 41-8. [DOI] [PubMed]

[21] D.A. Winter, F. Prince, J.S. Frank, C. Powell. Unified theory regarding $\mathrm{a} / \mathrm{p}$ and $\mathrm{m} / \mathrm{l}$ balance in quiet stance, Journal of Neurophysiology. 75(6) (1996) 2334-43. [DOI] [PubMed]

[22] A. Gebel, B. Lüder, \& U. Granacher, Effects of Increasing Balance Task Difficulty on Postural Sway and Muscle Activity in Healthy Adolescents, Frontiers in Physiology, 10 (2019) 1135. [DOI] [PubMed]

[23] D.C. Low, G.S. Walsh, \& M. Arkesteijn, Effectiveness of Exercise Interventions to Improve Postural Control in Older Adults: A Systematic Review and Meta-Analyses of Centre of Pressure Measurements, Sports Medicine (Auckland, N.Z.), 47(1) (2017) 101-112. [DOI] [PubMed]

\section{Funding}

No funding was received to carry out this study

\section{Authors Contribution}

Both the authors equally contributed to this work. Both the authors read and approved the final version of the manuscript.

\section{Ethics Approval}

IRB approval TWU protocol \# 20092

\section{Informed Consent}

Written consent obtained from the participants

\section{Conflict of interest}

The Authors have no conflicts of interest to declare that they are relevant to the content of this article.

\section{Does this article screened for similarity? Yes}

\section{About The License}

(c) The Author(s) 2021. The text of this article is open access and licensed under a Creative Commons Attribution 4.0 International License 